\title{
Estado actual y evolución del saneamiento y la depuración de aguas residuales en el contexto nacional e internacional
}

\author{
María Molinos Senante \\ Departamento de Estructura Económica, Facultad de Economía. \\ Universidad de Valencia \\ maria.molinos@uv.es \\ Francesc HernÁNDEZ SANCHO \\ Departamento de Estructura Económica, Facultad de Economía. \\ Universidad de Valencia \\ francesc.hernandez@uv.es \\ Ramón SALA GARRIDO \\ Departamento de Matemáticas para la Economía y la Empresa \\ Universidad de Valencia \\ ramon.sala@uv.es
}

Recibido: 13 de Marzo de 2012

Enviado a evaluar: 30 de Marzo de 2012

Aceptado: 24 de Abril de 2012

\section{RESUMEN}

El acceso al saneamiento y depuración de aguas residuales es un aspecto de suma importancia tanto desde el punto de vista ambiental como sanitario. Por ello, desde el Imperio Mesopotámico hasta la actualidad se han producido importantes avances en esta materia. Sin embargo, en la actualidad, en la mayoría de los países en vías de desarrollo el acceso al saneamiento continua siendo deficitario. Por otra parte, los países desarrollados, que sí disponen de sistemas adecuados de depuración de aguas residuales, se enfrentan al reto de mejorar la sostenibilidad de estos sistemas. En este trabajo se describe en profundidad el estado actual y evolución en el tiempo del saneamiento y depuración de aguas residuales a nivel internacional, europeo y español.

Palabras clave: Áreas sensibles a la eutrofización, depuración de aguas residuales, saneamiento, Directiva 91/271/CEE, reutilización de agua. 


\title{
Current status and development of sanitation and wastewater treatment in the national and international context
}

\begin{abstract}
Access to sanitation and wastewater treatment is an aspect of significant importance both from an environmental and health point of view. Therefore, since the Mesopotamian Empire until now there have been breakthroughs in this topic. However, at present, in most developing countries access to sanitation still deficit. On the other hand, developed countries that have adequate systems for wastewater treatment face the challenge of improving the sustainability of them. This work describes in depth the current state and evolution in time of the sanitation and wastewater treatment at International, European and Spanish level.
\end{abstract}

Key words: areas sensitive to eutrophication, wastewater treatment, sanitation, Directive 91/271/EEC, water reuse.

\section{État actuel et évolution de l'assainissement et épuration des eaux usées dans le contexte national et international}

\section{RÉSUMÉ}

L'Accès au traitement d'assainissement et de traitement des eaux usées est un aspect d'une importance primordiale tant du point de vue environnemental comme de la santé. Donc, depuis l'Empire Mésopotamien jusqu'à nos jours ont été percées dans ce domaine. Cependant, maintenant, dans la plupart des pays en développement l'accès à l'assainissement continue encore déficit. En outre, les pays développés, qui ont des égouts adéquats, font face au défi de l'amélioration de la durabilité de ces systèmes. Ce travail décrit en profondeur l'état actuel et l'évolution dans le temps, l'assainissement des eaux usées au niveau international, européen et espagnol.

Mots-clés: Zones sensibles à l'eutrophisation, des eaux usées, l'assainissement, Directive 91/271/CEE, réutilisation de l'eau.

\section{INTRODUCCIÓN}

El agua es un recurso esencial para el desarrollo de la vida. Así, los ecosistemas acuáticos proporcionan al conjunto de la sociedad una importante cuantía de beneficios tales como alimentos, medicinas, funciones recreativas, sumidero de residuos o captura de $\mathrm{CO}_{2}$. Sin embargo, el continuo crecimiento de la población, la industrialización, el aumento del nivel de vida y las inadecuadas prácticas de gestión han supuesto que en la actualidad nos enfrentemos a una importante crisis en el ámbito de los recursos hídricos tanto desde el punto de vista cuantitativo como cualitativo.

Todo ello ha supuesto que la mayoría de los países industrializados hayan desarrollado diversas normas con el objetivo de proteger los ecosistemas acuáticos y reflejar el verdadero valor del agua. Así, la Directiva Marco de Agua (Directiva 60/2000/CE) constituye una norma paradigmática con un marcado objetivo medioambiental pero haciendo uso de otro tipo de herramientas como son las de carácter económico. 
En este contexto, la adecuada gestión de las aguas residuales es un aspecto de suma importancia bajo dos perspectivas diferentes. Por una parte, tal y como la Directiva 91/271/CEE reconoce, los vertidos de aguas residuales urbanas constituyen, por su importancia, la segunda fuente de contaminación de medios acuáticos en forma de eutrofización. Por lo tanto, la recogida y depuración de aguas residuales previa a su vertido al medio receptor constituyen dos medidas básicas para prevenir el deterioro de los ecosistemas acuáticos. Por otra parte, el acceso al saneamiento está íntimamente relacionado con la calidad de vida y salud de las personas. Así, el inadecuado saneamiento es uno de los factores que más contribuye a la mortalidad de los niños menores de 5 años (Cumming, 2009). De hecho, la Organización Mundial de la Salud (OMS) estima que cada día mueren 1,8 millones de niños menores de 5 años por enfermedades relacionadas con la calidad del agua.

Por todo ello, el acceso al saneamiento se considera un derecho humano básico tal y como se recoge en los Objetivos de Desarrollo del Milenio (ODM) fijados en el año 2000 por los 192 países miembros de las Naciones Unidas. De forma más específica, se considera fundamental reducir a la mitad la proporción de personas sin acceso sostenible al agua potable y a servicios básicos de saneamiento en el año 2015. Así mismo, el acceso al saneamiento está relacionado con otros servicios esenciales como la educación. En este sentido, el Banco Mundial considera que a pesar del elevado coste del saneamiento, éste constituye la medida aislada con una mejor relación coste-eficacia para mejorar las condiciones sanitarias de los ciudadanos de los países en vías de desarrollo (World Bank, 2006).

En conclusión, se puede afirmar que el saneamiento es una medida básica que contribuye al logro de otros objetivos enmarcados en el ámbito de los ODM. Así, en los países en desarrollo, las inversiones en saneamiento son imprescindibles y deben dirigirse a: (i) reducir el volumen de agua residual generada a través de la adopción de medidas de prevención; (ii) implementar sistemas de alcantarillado; (iii) depurar el agua residual previamente a su vertido al medio ambiente utilizando tecnologías adecuadas para ello; (iv) en los casos que sea viable y seguro, reutilizar el agua reciclando en la medida de lo posible los nutrientes que contiene; y (v) proporcionar un entorno adecuado para el desarrollo de nuevas tecnologías y prácticas de gestión del agua residual (UNEP, 2010).

\section{ASPECTOS HISTÓRICOS DEL SANEAMIENTO Y DEPURACIÓN DE AGUAS RESIDUALES}

Si bien la importancia de la calidad del agua para abastecimiento urbano ha sido reconocida desde la antigüedad, lo cierto es que hasta el siglo XIX no se puso de manifiesto la necesidad de una adecuada gestión del agua residual como medio de protección de la salud pública (Lofrano y Brown, 2010). Durante siglos no se prestó atención al saneamiento y depuración de aguas residuales, provocando serios impactos negativos tanto sobre la salud de las personas como sobre el medio ambiente (Aiello et al., 2008). 
Los registros históricos muestran que el Imperio Mesopotámico (3500-2500 AC) fue la primera civilización que abordó formalmente los problemas relacionados con el saneamiento. Así, en las ruinas de Babilonia y Ur se han encontrado restos de casas que estaban conectadas a un precario sistema de alcantarillado para evacuar el agua residual (Jones, 1967). No obstante, también hay que destacar que la mayoría de las personas carecían de este sistema y vertían el agua residual directamente a las calles que periódicamente se cubrían con arcilla (Cooper, 2007).

La Civilización Hindú (1700-26 AC) estaba todavía más avanzada en la gestión del agua residual. En esta época, las casas estaban conectadas directamente a canales de desagüe y no estaba permitido verter el agua residual directamente a las calles sin un tratamiento previo que incluía como mínimo la sedimentación del agua residual (Wolfe, 1999).

La Civilización Griega (300 AC-500 DC) es considerada como la verdadera precursora de los sistemas de saneamiento "modernos". Los estudios arqueológicos han mostrado de forma unívoca que el origen de las prácticas modernas de gestión del agua residual data de la época de los griegos (Lofrano y Brown, 2010). La sociedad griega disponía de letrinas públicas que estaban conectadas a sistemas de alcantarillado que transportaban el agua residual junto con los pluviales fuera de la ciudad. Con posterioridad, esta agua era utilizada para regar y fertilizar los cultivos cercanos. Evidentemente, no todas las ciudades disponían de este complejo sistema, pero sí las más importantes como por ejemplo Atenas, Thasos o Pergamon entre otras (TolleKastenbein, 2005).

Si bien los romanos no inventaron el sistema de alcantarillado, lo cierto es que fue altamente perfeccionado por ellos. Así, el Imperio Romano gestionaba el ciclo urbano del agua desde el abastecimiento de agua potable hasta la recogida del agua residual utilizando un doble sistema de tuberías (Jones, 1967). Entre todas las redes de alcantarillado construidas por los romanos destaca por su longitud y robustez (una parte de ella sigue utilizándose en la actualidad) la llamada "Cloaca Maxima" en Roma (Lofrano y Brown, 2010). En todas las ciudades más importantes del Imperio Romano se han encontrado restos de redes de canales para el transporte de agua mostrando una amplia diversidad de técnicas constructivas en función de la geología, pendiente y otras variables topológicas.

El final del Imperio Romano marcó el comienzo de los llamados años negros del saneamiento que duraron más de 1000 años (476 -1800 DC). Durante esa época se abandonó la cultura del agua como fuente de salud y bienestar. En lo que se considera una regresión histórica sin precedentes, el agua residual era vertida directamente sin ningún tipo de tratamiento constituyendo un importante foco de enfermedades (Sori, 2001). Durante ese largo periodo, muy pocas casas disponían de instalaciones de tipo sanitario siendo la práctica habitual el vertido del agua residual directamente a las calles. A partir del siglo XIX, encontramos algunas excepciones a esta práctica habitual por ejemplo en algunas ciudades del norte de Italia siendo Florencia una de las más avanzadas en este ámbito.

El fuerte proceso de industrialización y el crecimiento de las ciudades experimentado durante el siglo XVIII puso de manifiesto la importancia de una adecuada ges- 
tión del agua residual (Tarlow, 2007). Por ejemplo, en 1865 Londres disponía de una red de alcantarillado que vertía el agua residual directamente al río Támesis. Las principales ciudades italianas en el año 1899 contaban con sistemas de alcantarillado (Giovannini, 1996).

La gran revolución en el ámbito del saneamiento y depuración de aguas residuales tuvo lugar durante el siglo XX. Un importante hito se produjo en 1912 cuando la "Royal Commission on Sewage Disposal" fijó las normas y ensayos que se debían aplicar para caracterizar el efluente de las instalaciones de depuración. A partir de ese año, las principales ciudades europeas comenzaron a construir estaciones depuradoras de aguas residuales. No obstante tanto la Primera como la Segunda Guerra Mundial retrasaron la puesta en funcionamiento de este tipo de instalaciones (Seeger, 1999).

\section{ESTADO ACTUAL Y EVOLUCIÓN DEL SANEAMIENTO A NIVEL MUNDIAL}

En los últimos veinte años se han realizado importantes esfuerzos a nivel mundial para incrementar el porcentaje de población con acceso a servicios de saneamiento básicos. A pesar de ello, en la actualidad todavía hay unos 2.600 millones de personas que carecen de estos servicios, es decir, más de un tercio de la población mundial no dispone de acceso al saneamiento.

La Figura 1 muestra que existen importantes divergencias entre regiones en relación al acceso a este servicio básico. Mientras que en los países desarrollados se considera que toda la población (99\%) dispone de un adecuado saneamiento, en los países en vías de desarrollo, el porcentaje se reduce considerablemente siendo sólo del $52 \%$.

Figura 1. Porcentaje de población con acceso a servicios de saneamiento. Año 2008

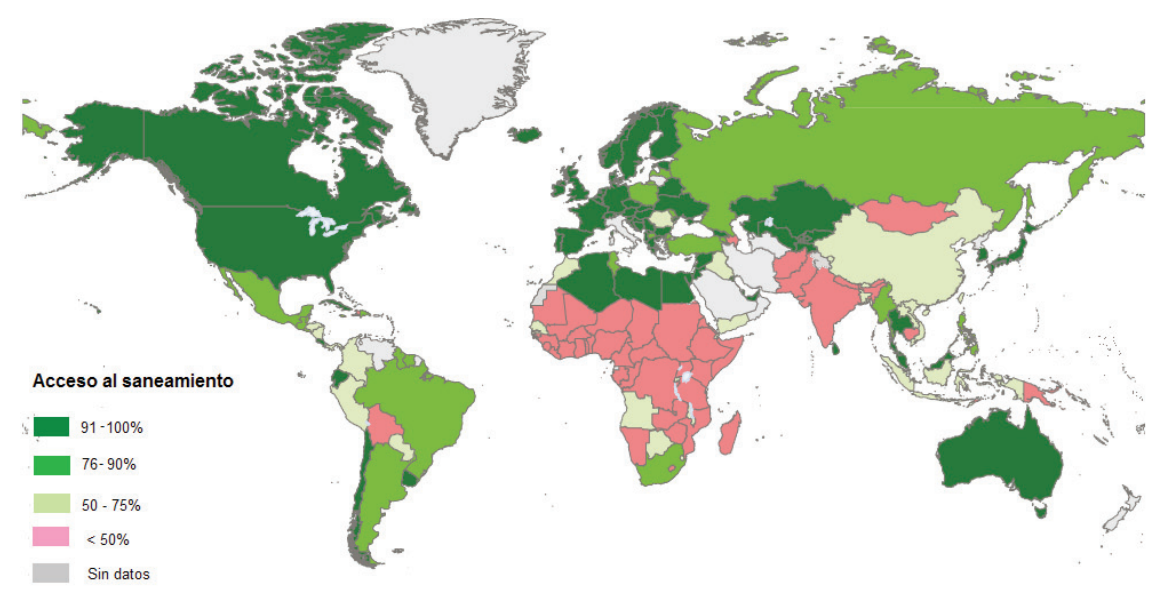

Fuente: Adaptado de WHO-UNICEF (2010) 
Si centramos el análisis en la distribución regional (Figura 2), se observa que es en el Sureste asiático donde el número de personas sin acceso al saneamiento es mayor. No obstante, también son de destacar las regiones del Este de Asia y África Subsahariana. Una agrupación por continentes muestra que Asia y África concentran el $72 \%$ y $22 \%$ respectivamente de todas las personas sin acceso al saneamiento. Por otra parte, incluso en los países desarrollados hay 15 millones de personas que carecen de servicios de saneamiento.

Figura 2. Millones de personas sin acceso a servicios de saneamiento. Año 2008.
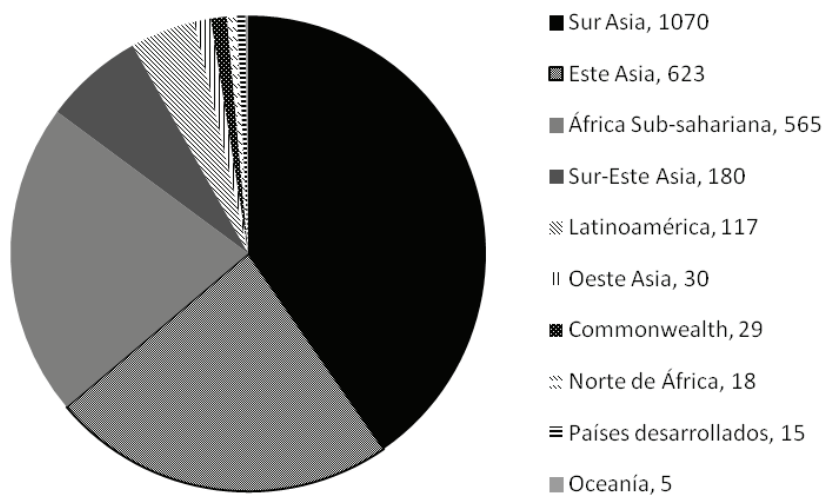

Fuente: Adaptado de WHO-UNICEF (2010)

En cuanto a los progresos realizados desde el año 1990 hasta el 2008, la Figura 3 muestra que hay importantes disparidades entre regiones. Así, el Norte de África, el Sureste asiático y el Este de Asia han incrementado significativamente el porcentaje de población con acceso al saneamiento mientras que en Oceanía se ha producido un retroceso.

Figura 3. Progreso desde 1990 a 2008 en el acceso al saneamiento (\%)

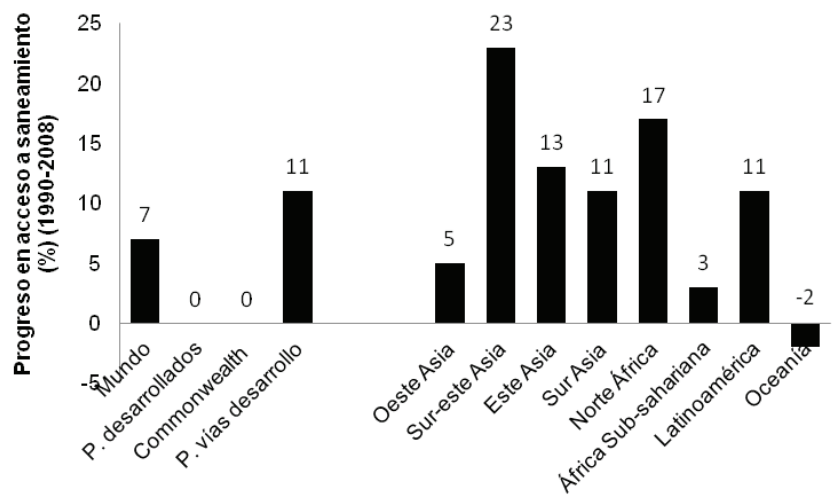

Fuente: Adaptado de WHO-UNICEF (2010) 
Las cifras mostradas en las Figuras 1 y 2 indican que en materia de acceso al saneamiento hay una importante disparidad entre las regiones desarrolladas y las no desarrolladas. Así mismo, tal y como se muestra en la Figura 4, esta disparidad también se produce entre las zonas urbanas y rurales. En el año 2008, 794 millones de personas sin acceso a saneamiento vivían en áreas urbanas mientras que $1856 \mathrm{mi}$ llones lo hacían en áreas rurales. Estas cifras indican que 7 de cada 10 personas sin acceso al saneamiento viven en áreas rurales. De los 1300 millones de personas que accedieron al saneamiento durante el periodo de 1990 a 2008, el 64\% de ellas vivían en áreas urbanas. No obstante, a pesar de que las áreas urbanas están mejor servidas que las áreas rurales, se enfrentan al problema del continuo crecimiento de la población urbana (WHO-UNICEF, 2010).

Figura 4. Porcentaje de población urbana y rural con acceso a saneamiento. Año 2008

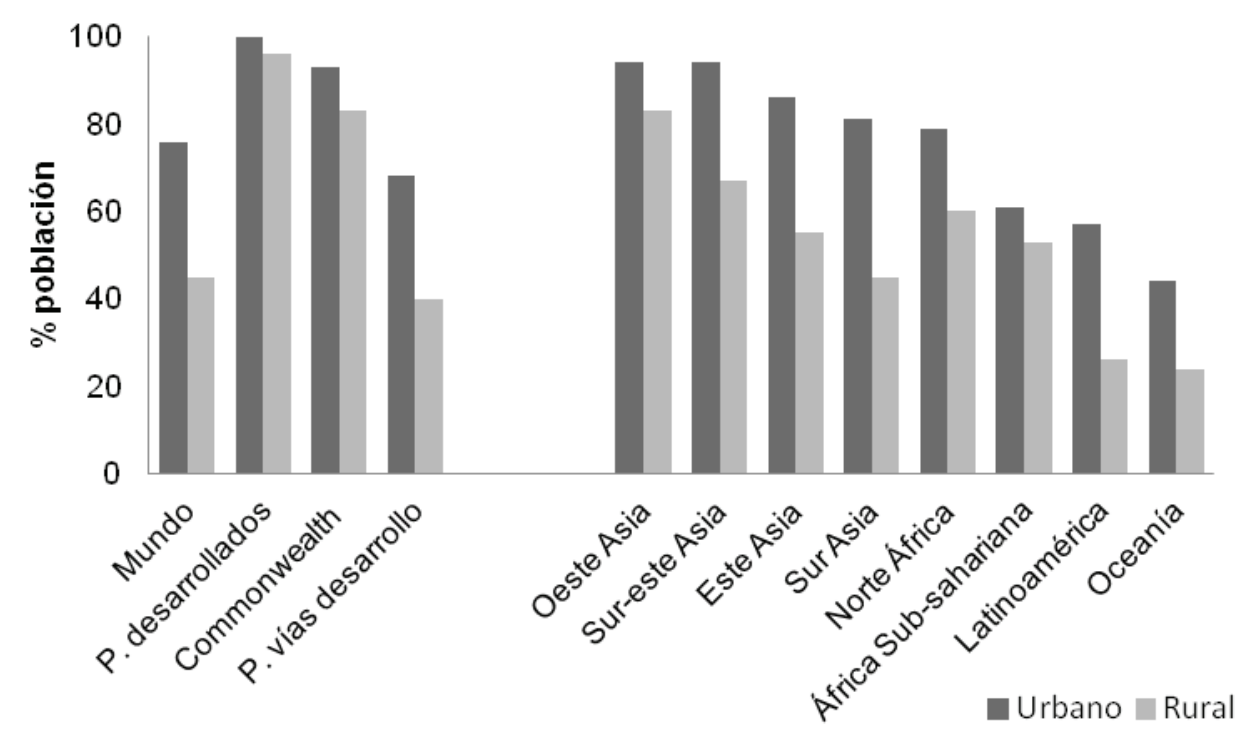

Fuente: Adaptado de WHO-UNICEF (2010)

$\mathrm{Si}$ analizamos este tipo de discrepancia (rural-urbano) por regiones, se observa que son especialmente significativas en Latinoamérica, en el Sur de Asia y Oceanía. Por otra parte, dado que en el África Sub-sahariana, en el Sur de Asia y en Oceanía la mayoría de la población vive en áreas rurales, las divergencias en estas regiones son especialmente importantes en relación al número de personas afectadas.

Tal y como hemos descrito en la introducción, el acceso a los servicios básicos de saneamiento es uno de los Objetivos del Milenio de las Naciones Unidas para el año 2015. De forma más específica, el objetivo es reducir el porcentaje de población sin acceso al saneamiento al $23 \%$ de la población mundial. Sin embargo, teniendo en cuenta la evolución actual, este objetivo no se alcanzará ya que las proyecciones in- 
dican que en el año 2015, un 36\% de la población mundial todavía carecerá de acceso al saneamiento.

Una vez que el estado actual y la evolución del acceso a los servicios básicos de saneamiento ha sido descrito a nivel mundial, en la siguiente sección, analizaremos con más detalle los datos correspondientes a los países miembros de la Unión Europea (UE).

\section{ESTADO ACTUAL Y EVOLUCIÓN DEL SANEAMIENTO EN LA UNIÓN EUROPEA}

La Directiva 91/271/CEE de 21 de mayo de 1991, sobre el tratamiento de aguas residuales urbanas supuso una importante mejora en el saneamiento y depuración de aguas residuales en los Estados Miembro de la UE (EM). La citada Directiva establece las medidas necesarias que los EM han de adoptar para garantizar que las aguas residuales urbanas reciben un tratamiento adecuado antes de su vertido. De forma resumida, la Directiva establece dos obligaciones claramente diferenciadas. En primer lugar las "aglomeraciones urbanas" deben disponer, según los casos, de sistemas de colectores para la recogida y conducción de las aguas residuales y, en segundo lugar, se prevén distintos tratamientos a los que deberán someterse dichas aguas antes de su vertido a las aguas continentales o marinas. Así mismo, en la determinación de los tratamientos a que deberán someterse las aguas residuales antes de su vertido, se tienen en cuenta las características del emplazamiento donde se producen. De acuerdo con esto, los tratamientos serán más o menos rigurosos según se efectúen en zonas calificadas como sensibles o no sensibles a la eutrofización.

A pesar de que la Directiva 91/271/CEE es de obligatorio cumplimiento para los 27 EM, el Sistema Europeo de Información sobre el Agua (Water Information System for Europe, WISE) únicamente contiene información relativa al saneamiento y depuración de aguas para 18 países europeos: Alemania, Austria, Bélgica, Chipre, Dinamarca, Estonia, Eslovaquia, Eslovenia, Finlandia, Francia, Hungría, Letonia, Lituania, Luxemburgo, Países Bajos, Portugal, Rumanía y Suecia. La razón de ello, es que sólo estos países han presentado a la Comisión Europea los informes para valorar el grado de aplicación de la Directiva en la forma que fueron solicitados. A fecha de Diciembre de 2006, estos 18 países contaban con 13.734 aglomeraciones urbanas de más de 2.000 habitantes equivalentes ${ }^{1}$ (he).

Tal y como se muestra en la Figura 5 , el 93\% de la carga contaminante generada en la UE-18 es recogida mediante sistemas de alcantarillado adecuados. Un $87 \%$ de la carga contaminante es depurada mediante los denominados tratamientos secundarios de los cuales el 78\% funcionan adecuadamente. Finalmente, otros tratamientos más avanzados se utilizan para tratar un $72 \%$ de la carga contaminante siendo el $65 \%$ el porcentaje que funcionan de forma adecuada. Estas cifras indican que la mayoría

${ }^{1}$ Un habitante equivalente representa la carga orgánica biodegradable con una demanda biológica de oxígeno de cinco días $\left(\mathrm{DBO}_{5}\right)$, de 60 gramos de oxígeno por día. 
del agua residual generada llega a las estaciones depuradoras de aguas residuales (EDARs) donde reciben un tratamiento adecuado.

Figura 5. Carga contaminante tratada en aglomeraciones mayores de 2.000 he.

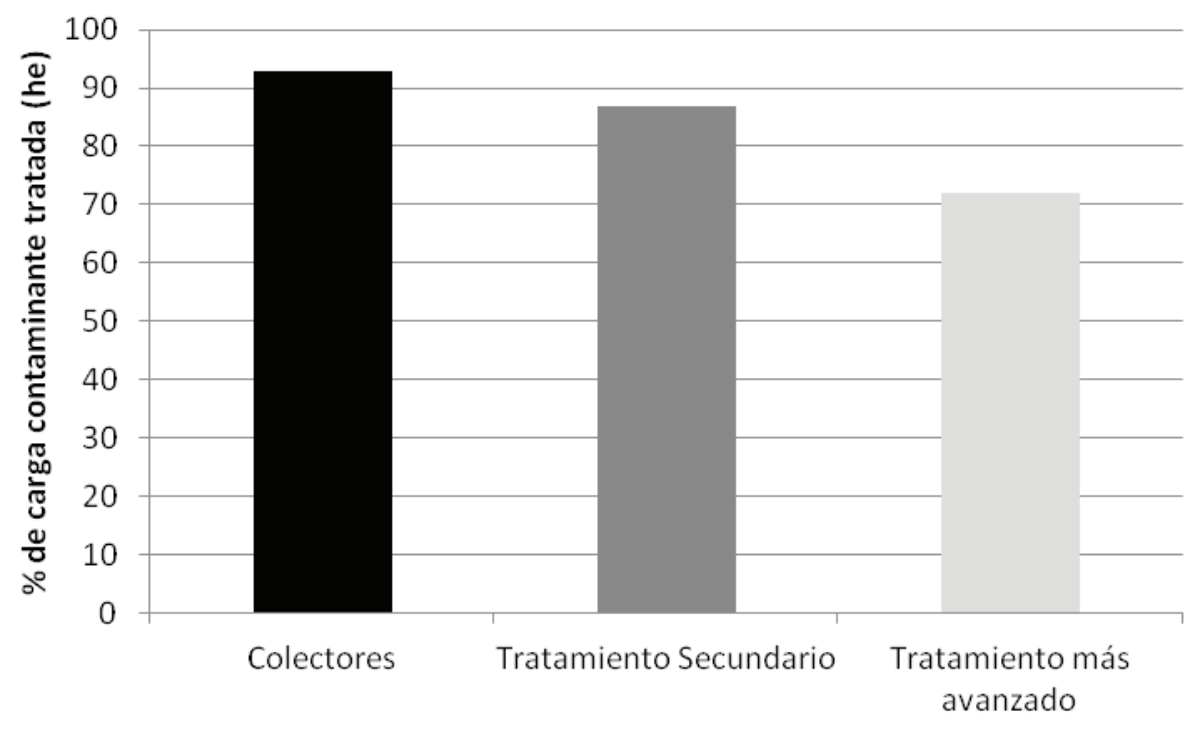

Fuente: Adaptado de WISE (2011)

Según el artículo 5(1) de la Directiva 91/271/CEE, los EM tienen que identificar las áreas sensibles a la eutrofización y revisar dicha identificación cada 4 años -Artículo 5(6). Sin embargo, el Artículo 5(8) de esta misma Directiva establece que un EM no deberá designar zonas sensibles cuando depure todas las aguas residuales generadas en su territorio según el criterio establecido para dichas zonas. En relación a los tratamientos más avanzados a aplicar cuando el vertido se produce a zonas sensibles, la Directiva permite dos opciones. La primera de ellas, (Artículo $5(2,3)$ ) hace referencia a alcanzar una determinada calidad de efluente en instalaciones con capacidad de tratamiento superior a 10.000 he. La segunda opción (Artículo 5(4)) no requiere una determinada calidad en el efluente sino alcanzar como mínimo un $75 \%$ de reducción en la carga de nitrógeno y fósforo en todas las instalaciones.

Con fecha de 31 de Diciembre de 2006, diez EM se habían acogido al Artículo 5(8), es decir, aplicaban tratamientos más exigentes en todo su territorio: Austria, Dinamarca, Estonia, Finlandia, Letonia, Lituania, Luxemburgo, Países Bajos, Polonia y Rumania. Por otra parte, aunque Bélgica, Eslovaquia y Suecia no se acogen al Artículo 5(8) han identificado todas sus masas de agua como sensibles a la eutrofización. El resto de países (Alemania, Bulgaria, Chipre, Eslovenia, España, Francia, Hungría, Italia, Malta, Portugal y Reino Unido) han identificado las masas de agua que son 
sensibles a la eutrofización. Con fecha de Diciembre de 2008, el 68\% del territorio de la EU-27 había sido definido como área sensible a la eutrofización.

Las aglomeraciones de los 18 EM estudiados generan un volumen de carga contaminante de 317,7 millones de he. La carga asociada a las zonas sensibles que se acogen al Artículo 5(4) es 145 millones de he incluyendo 4.839 aglomeraciones. En relación a las zonas sensibles que aplican el Artículo 5(2,3) la carga es de 115 millones de he y el número de aglomeraciones es de 6.306. Finalmente, en las 2.589 aglomeraciones que conforman las zonas no sensibles, se genera una carga contaminante de 54 millones de he. La Figura 6 muestra las infraestructuras de recogida y depuración de aguas residuales en función del emplazamiento donde se realiza el vertido: zonas sensibles acogidas al Artículo 5(4), zonas sensibles acogidas al Artículo $5(2,3)$ y zonas no sensibles a la eutrofización.

Figura 6. Carga contaminante tratada en aglomeraciones mayores de 2.000 he en función de la zona de vertido

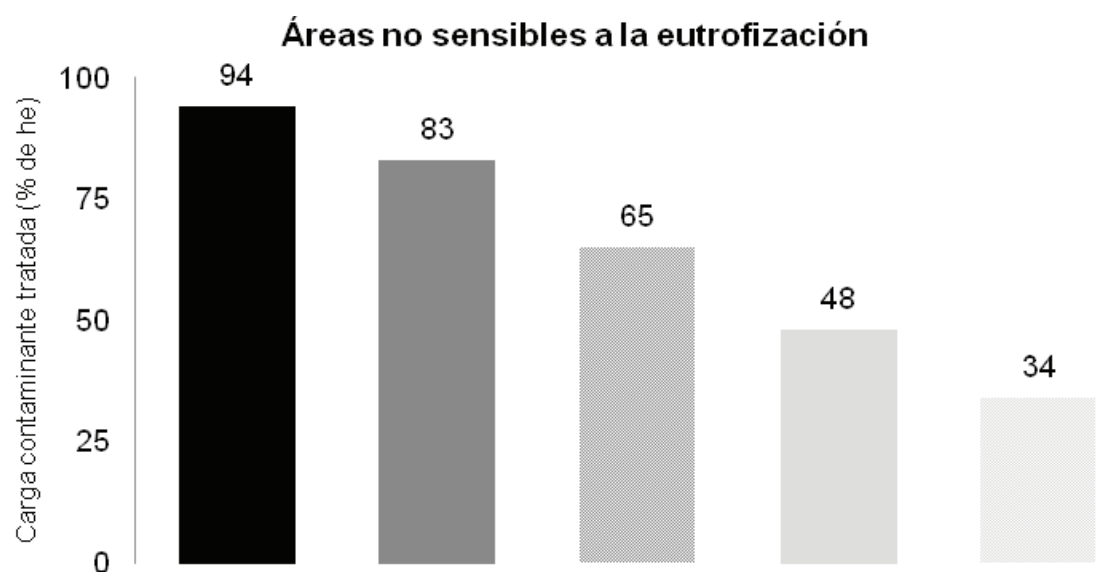

Áreas sensibles a la eutrofización Art $5(2,3)$

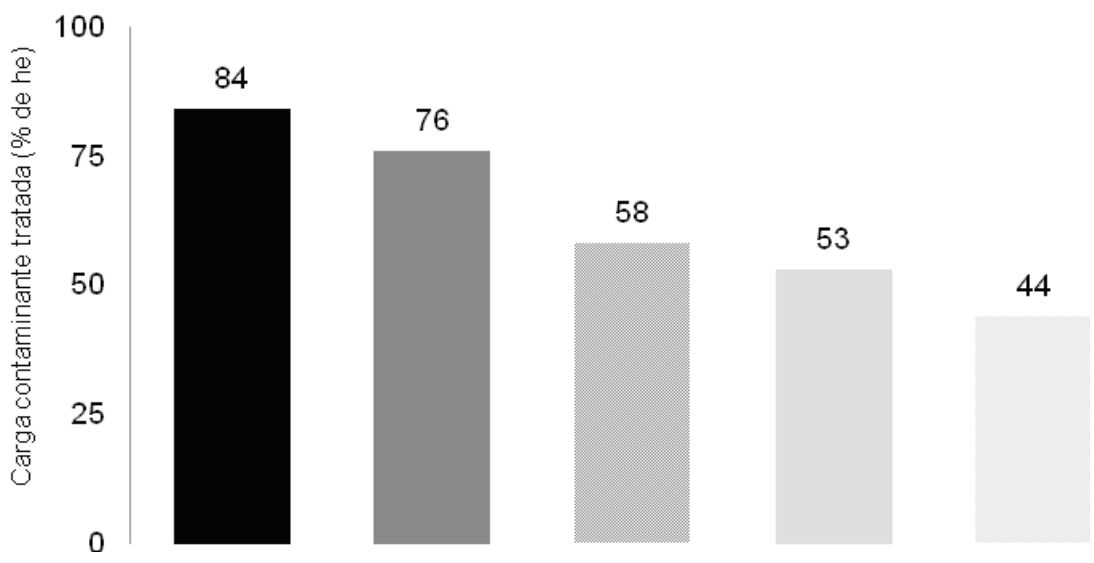




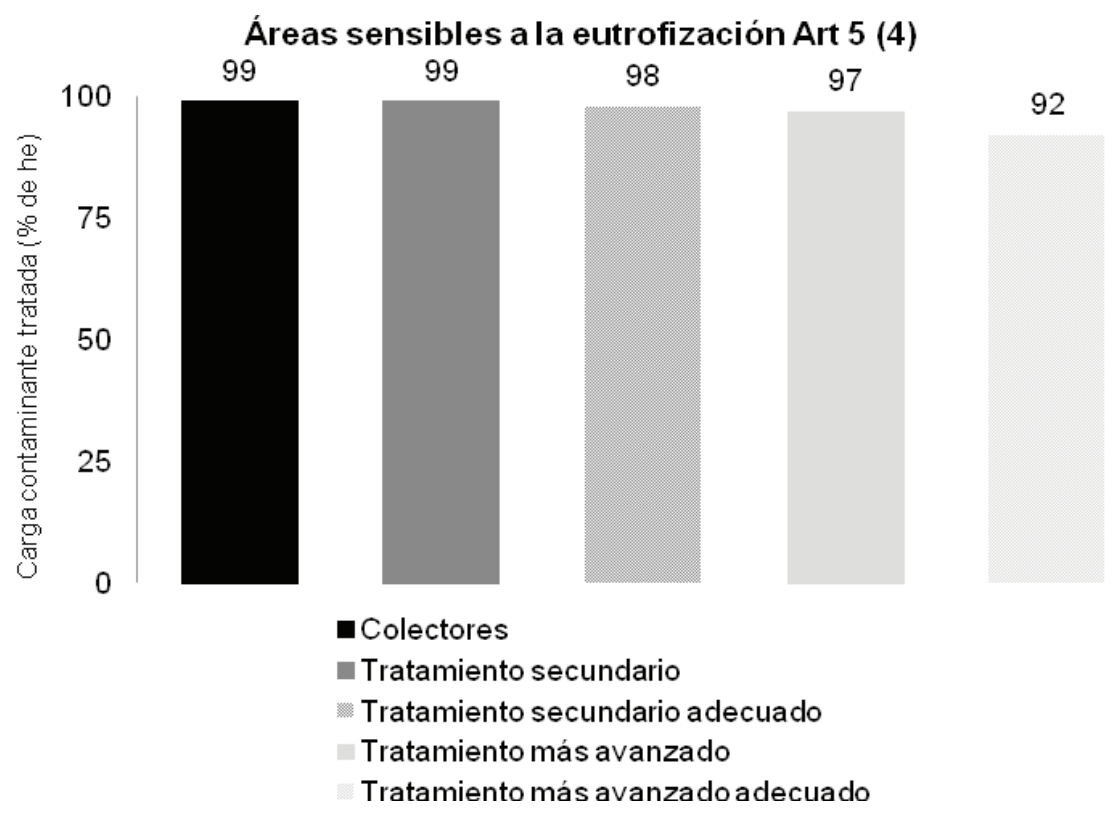

Fuente: Adaptado de WISE (2011)

Se observa que las áreas que se acogen a lo dispuesto en el Artículo 5(4) disponen de más y mejoras infraestructuras de tratamiento de aguas residuales que aquellas que se acogen al Artículo 5(2,3). Es de destacar que en las zonas no sensibles a la eutrofización no todas las aguas residuales son recogidas ni son depuradas con tratamiento de tipo secundario. Sin embargo, a pesar de que no es una obligación establecida por la Directiva, algunas de las instalaciones disponen de tratamientos más avanzados. En relación a las zonas sensibles regidas por el Artículo 5(2,3), sólo el 84\% dispone de sistemas de recogida de agua residual, el $76 \%$ son depuradas con tratamiento de tipo secundario y sólo el 53\% reciben tratamiento más avanzado. Una de las razones que justifica el bajo porcentaje de carga contaminante depurada con tratamientos secundarios en las áreas que se rigen por el Artículo 5(2,3) es el hecho de que a muchos países se les ha permitido una moratoria en la implementación de la Directiva 91/271/ CEE en relación a las áreas sensibles.

Si ampliamos la escala espacial y consideramos otros países europeos que no forman parte de la UE, podemos observar importantes diferencias entre ellos en relación al porcentaje de su población cuya agua residual es tratada en EDARs (Figura 7). Si bien es de destacar que la información proporcionada en la Figura 7 corresponde a distintos años en función de la disponibilidad de datos. 
Figura 7. Población (millones) conectada a EDARs en países europeos

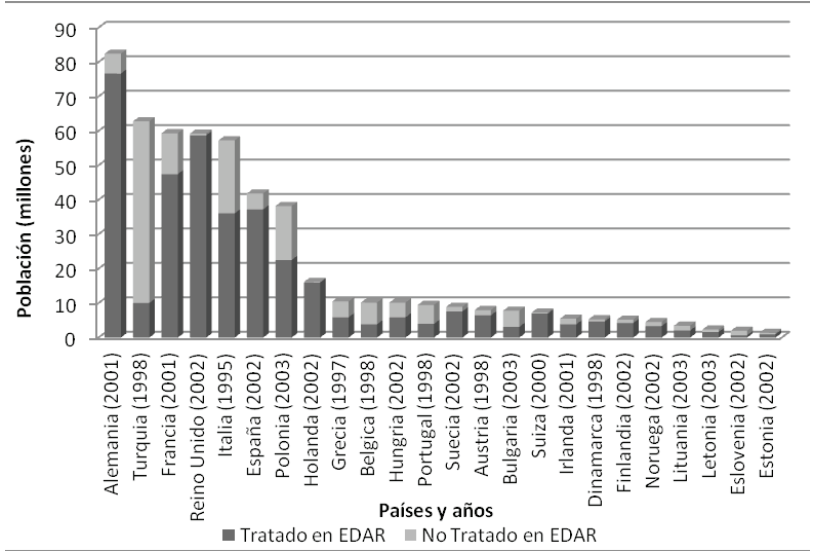

Fuente: Adaptado de WISE (2011)

En un análisis más detallado, la Figura 8 muestra el porcentaje de cada tipo de tratamiento (primario, secundario y terciario). Se observa que hay algunos países como Alemania, Países Bajos, Suecia, Austria, Dinamarca, Finlandia, Noruega, Estonia y Chipre en los que la mayoría del agua residual se depura haciendo uso de tratamientos de tipo terciario. Por el contrario, en otros países como Grecia, Irlanda, Lituania e Islandia la mayor parte del agua residual únicamente se depura con tratamientos primarios. Así mismo, es de destacar que ninguna de las EDARs de Turquía e Islandia cuentan con tratamientos de tipo avanzado o terciario.

Figura 8. Porcentaje de población conectada a EDAR según el tipo de tratamiento

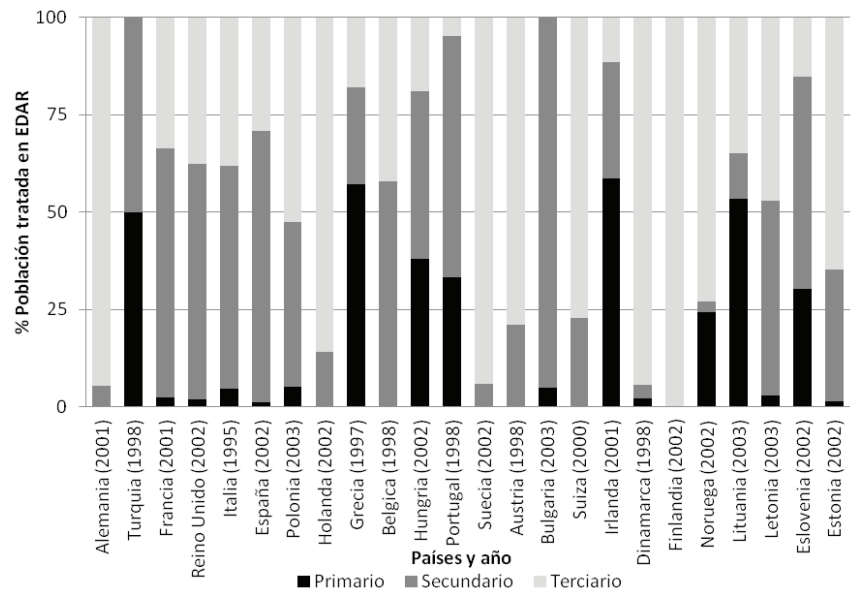

Fuente: Adaptado de WISE (2011) 
Las cifras mostradas previamente nos informan sobre el estado del saneamiento y depuración de aguas residuales en Europa con fecha de Diciembre de $2007^{2}$. Con el objetivo de describir los progresos realizados en esta materia, la Figura 9 muestra la evolución temporal desde 1990 a 2007 en el porcentaje de población con servicio al tratamiento de aguas residuales por regiones europeas.

Figura 9. Variación regional en el servicio de depuración entre el año 1990 y 2007

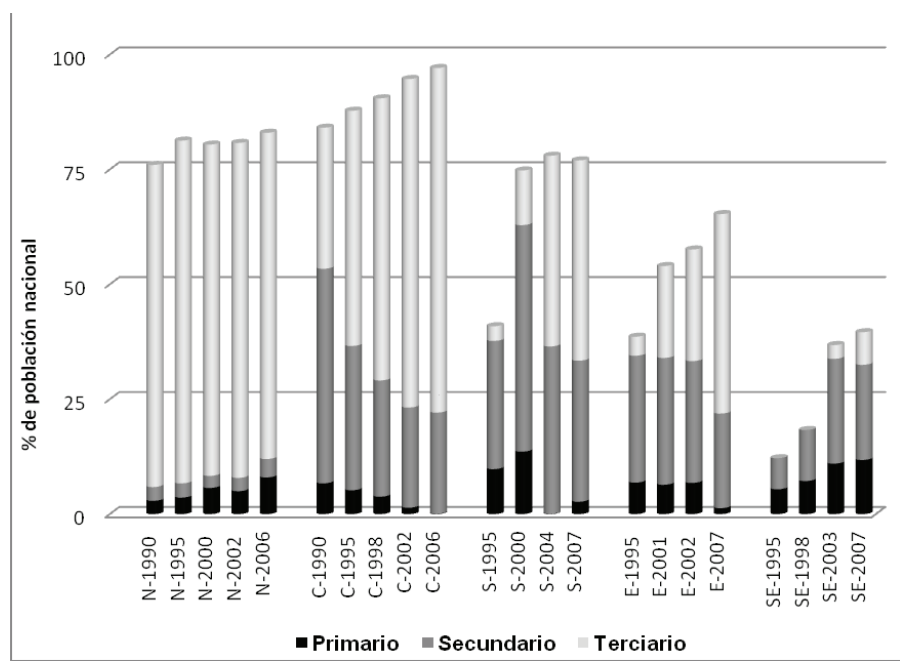

Fuente: Adaptado de WISE (2011)

La región categorizada como Norte engloba a Noruega, Suecia, Finlandia e Islandia. En esta región el porcentaje de población con acceso al tratamiento de agua residual ha incrementado desde el $76 \%$ en 1990 hasta el $83 \%$ en 2006 . Esta mejora se ha producido en los tres niveles de tratamiento. Así mismo, es de destacar que en 2006 el $71 \%$ de la población disponía de tratamiento terciario. La denominación región Central incluye a Austria, Dinamarca, Reino Unido, Países Bajos, Alemania, Suiza, Luxemburgo e Irlanda. En esta área entre 1990 y 2006, el porcentaje de población con acceso al tratamiento de agua residual se ha incrementado aproximadamente en un $10 \%$. Si bien, en esta región los esfuerzos se han focalizado en incrementar el porcentaje de población con tratamiento terciario (el aumento en este tratamiento ha sido de más del 40\%). La región del Sur engloba a Chipre, Grecia, Francia, Malta, España y Portugal. En este caso, el mayor incremento se produjo durante el periodo comprendido entre 1995 y 2000 en el que el aumento se cifró en un 34\%. Entre 2000 y 2004 los esfuerzos

${ }^{2} \mathrm{Se}$ trata de la información disponible más actual tomando como referencia el $5^{\circ}$ Informe de la Comisión Europea en relación a la implementación de la Directiva 91/271/CEE. 
se centraron en incrementar el porcentaje de población con tratamiento terciario. Desde 2004 a 2007 la situación se ha mantenido prácticamente constante. La denominada región del Este incluye a la República Checa, Estonia, Hungría, Letonia, Lituania, Polonia, Eslovaquia y Eslovenia. La Figura 9 muestra que durante el periodo de tiempo estudiado, el incremento ha sido de un $25 \%$. Sin embargo, dado que en 1990 la situación era muy precaria, el porcentaje de población con acceso al tratamiento de aguas residuales continua siendo bajo (65\%). Finalmente, la región categorizada como Sur-Este engloba a Bulgaria, Rumania y Turquía. En estos países, el porcentaje de población conectada a sistemas de depuración de agua residual en 2007 era muy bajo (40\%). Por lo tanto, en un futuro cercano deberán realizarse importantes esfuerzos en estos países en materia de saneamiento.

Tal y como se ha descrito previamente, la Comisión Europea cuando elaboró el $5^{\circ}$ Informe sobre la implementación de la Directiva 91/271/CEE correspondiente al año 2007 no disponía de información sobre todos los EM. Entre ellos se encontraba España que no proporcionó la información a la Comisión Europea en el formato adecuado hasta Abril de 2008. Por ello, con el objetivo de conocer el estado actual y evolución del saneamiento y depuración de aguas residuales en España, en la siguiente sección se analiza con detalle la información disponible para nuestro país.

\section{ESTADO ACTUAL Y EVOLUCIÓN DEL SANEAMIENTO EN ESPAÑA}

A fecha de Diciembre de 2011 en España había 2.412 aglomeraciones urbanas de más de 2.000 he lo que implica una carga contaminante total de 71.784 .819 he. En la Figura 10 se observa la distribución territorial de estas aglomeraciones y de las áreas definidas como sensibles a la eutrofización. En algunas zonas parece que no haya aglomeraciones urbanas. Si las hay, lo que ocurre es que son inferiores a 2.000 he correspondiéndose a pequeños municipios en áreas rurales. Así mismo, también se identifican las áreas de mayor concentración urbana que se corresponden con las zonas donde las necesidades de inversión y mantenimiento en instalaciones de gestión de aguas residuales son mayores (SIA, 2011). De todas las aglomeraciones urbanas identificadas, aproximadamente unas 650 tienen una población superior a 15.000 he representando el $87 \%$ del total de la carga generada en España. A fecha de 31 de Diciembre de 2005, el 77\% de estas aglomeraciones cumplían los requerimientos establecidos por la Directiva 91/271/CEE (MARM, 2006). Por otra parte, se han definido 837 zonas sensibles a la eutrofización lo que representa un $31 \%$ del territorio nacional. 
Figura 10. Aglomeraciones urbanas y áreas sensibles

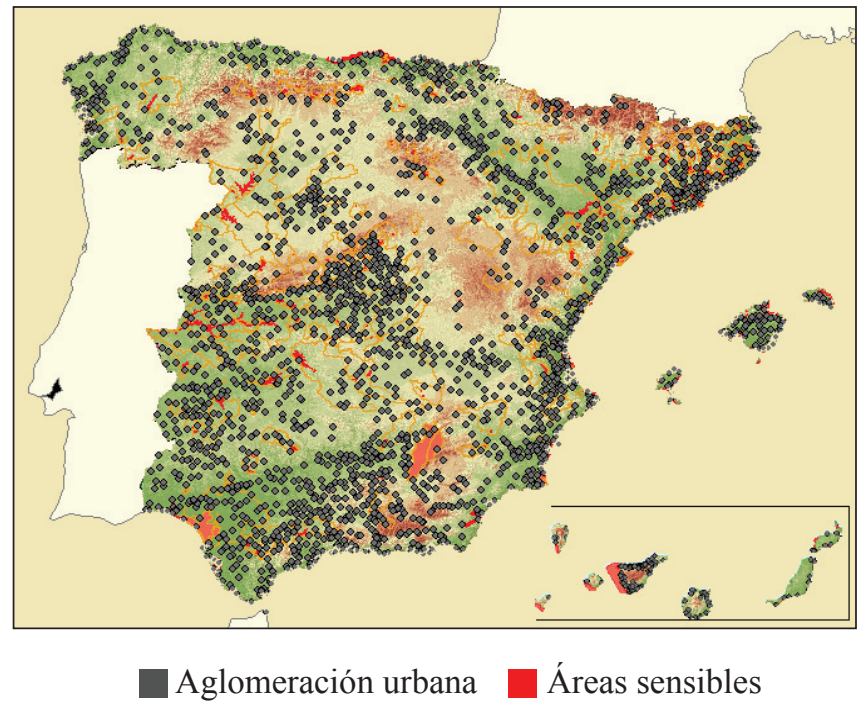

Fuente: Adaptado de SIA (2011)

En un análisis detallado por Comunidades Autónomas (CC.AA), la Figura 11 muestra el volumen de agua residual total tratado por cada una de ellas ( $\mathrm{hm}^{3} /$ día) así como por habitante ( $\mathrm{m}^{3} /$ día) para el año 2009.

Figura 11. Agua residual tratada por regiones (total y per cápita)

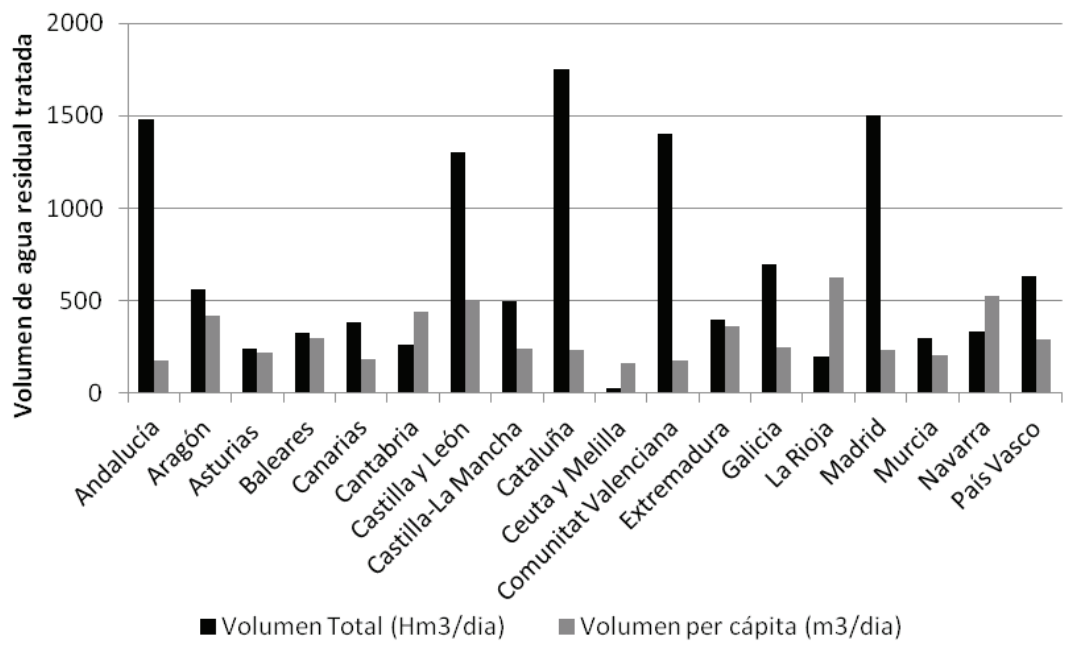

Fuente: Elaboración propia a partir de INE (2012) 
Se observa que la comunidad autónoma donde se trata un mayor volumen de agua residual es Cataluña seguida de Madrid y Andalucía. Sin embargo, cuando se tiene en cuenta la población de cada región, la ordenación es distinta. Bajo este criterio, son Castilla-León, Navarra y Aragón las tres regiones que tratan mayor volumen per cápita de agua residual.

En relación a la evolución temporal en el volumen de agua residual tratada, la Figura 12 muestra que durante los años 2000 a 2009 se ha producido una mejora significativa. De forma más específica, el volumen total de agua residual tratada en España en el año 2000 era de 7,7 millones de m³ $^{3}$ día mientras que en 2009 fue de 12,4 millones de $\mathrm{m}^{3} /$ día (aumento del $60 \%$ ). Además, esta mejora se observa en todas las CC.AA. Es de destacar el excepcional crecimiento producido en las regiones de Castilla-León, Valencia y Madrid.

Figura 12. Evolución en el volumen de agua residual tratada por regiones (2000-2009)

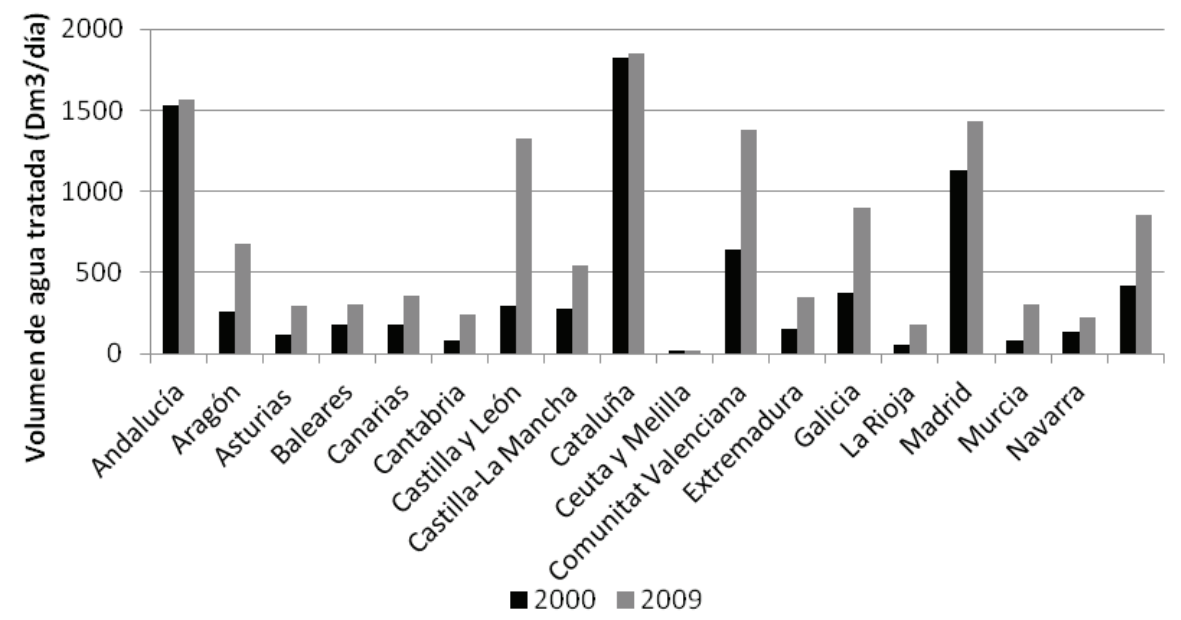

Fuente: Elaboración propia a partir de INE (2012)

En el contexto de la gestión de recursos hídricos y teniendo en cuenta los problemas endémicos de escasez de agua existentes en determinadas regiones españolas, en los últimos años el uso de fuentes de agua no convencionales, como son la reutilización de agua regenerada y la desalación de agua de mar, ha adquirido una gran importancia. Este suministro de agua es especialmente relevante en las áreas del sureste peninsular y los archipiélagos tanto Balear como Canario dada la escasez e irregularidad de los recursos hídricos convencionales en estos territorios.

Como prueba de la importancia creciente de la reutilización de aguas residuales, el Ministerio de la Presidencia aprobó a propuesta de los Ministerios de Medio Ambiente, de Agricultura, Pesca y Alimentación y de Sanidad y Consumo, el Real Decreto 1620/2007, de 7 de Diciembre, por el que se establece el régimen jurídico de 
la reutilización de aguas depuradas en España. Entre otros aspectos, la norma define el concepto de reutilización, introduce la denominación de aguas regeneradas, determina los requisitos necesarios para llevar a cabo la actividad de utilización de estas aguas y los procedimientos para obtener la concesión exigida en la Ley. Además, recoge los criterios de calidad mínimos obligatorios exigibles para la utilización de las aguas regeneradas según los usos. En todos los casos, el Organismo de Cuenca solicitará a las autoridades sanitarias un informe que tendrá carácter vinculante. Por otro lado, se prohíben determinados usos que presentan riesgos para la salud humana y el medio ambiente.

El volumen total de agua regenerada reutilizada en España durante el año 2009 fue $1.464 .261 \mathrm{~m}^{3} /$ día. La Figura 13 muestra la distribución de este volumen por CC.AA. Se observa que más de un tercio del total de agua es reutilizada en la región de Valencia seguida de las regiones de Murcia y Andalucía. Por otra parte, hay regiones como Navarra, Cantabria o Asturias que por sus condiciones climáticas no necesitan hacer uso de esta fuente alternativa de recursos hídricos. Considerando el volumen de agua reutilizada per cápita (Figura 13), Murcia es la región con mayor tasa de reutilización de agua $\left(65 \mathrm{hm}^{3} /\right.$ año per cápita), seguida de las Islas Baleares (41 hm³/año per cápita) y la región de Valencia $\left(21 \mathrm{hm}^{3} /\right.$ año per cápita).

Figura 13. Agua regenerada reutilizada por regiones (total y per cápita)

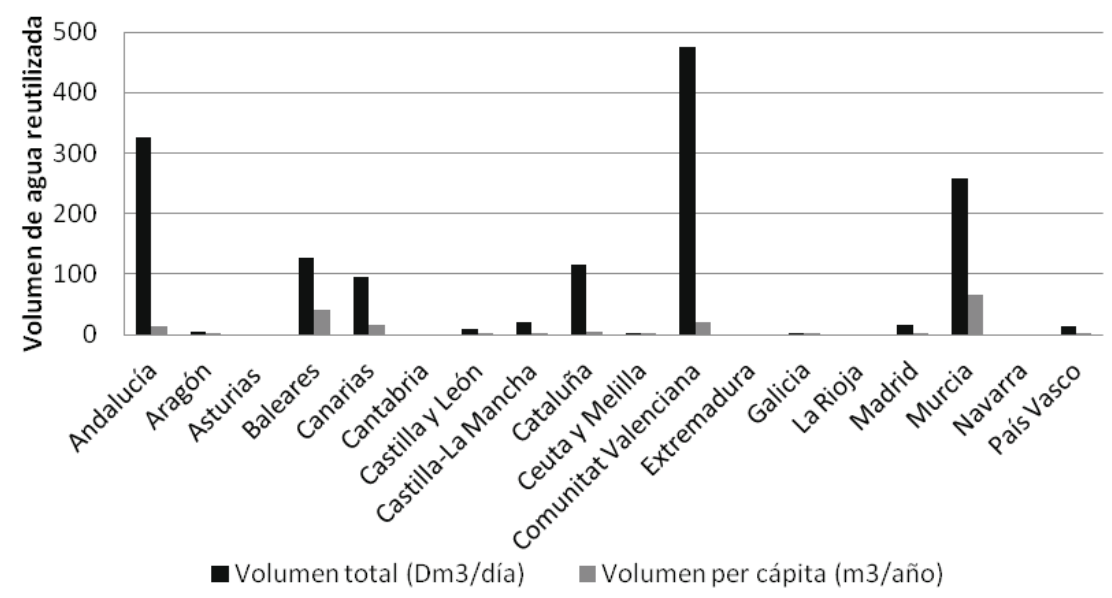

Fuente: Elaboración propia a partir de INE (2012)

En relación a los usos del agua regenerada, la Figura 14 muestra que en España el uso fundamental es el riego agrícola. El segundo uso más importante es el denominado "otros usos" que hace referencia a usos ambientales, principalmente en relación al mantenimiento y regeneración de humedales. También es de destacar que el 12\% del agua regenerada se usa para el riego de campos de golf. El resto de usos son minoritarios ya que representan un porcentaje inferior al 5\% del total de agua regenerada. 
Figura 14. Uso del agua regenerada reutilizada en España
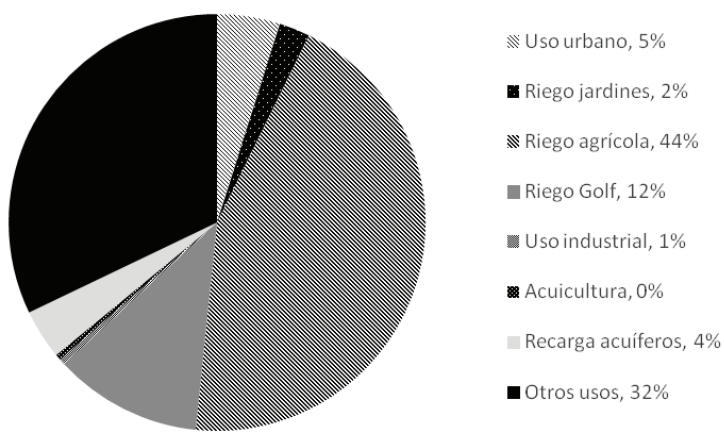

Fuente: Adaptado de SIA (2011)

Para finalizar el análisis sobre el estado y evolución del saneamiento y depuración en España, la Figura 15 muestra los costes de inversión relacionados con la recogida y tratamiento de agua residual. Se observa que algunas CC.AA (Asturias, Islas Canarias, Cantabria y País Vasco) realizaron la mayoría de las inversiones en el año 2000 lo que les permitió alcanzar los requerimientos exigidos por la Directiva 91/271/CEE en el marco temporal adecuado. Un segundo grupo de regiones realizaron las inversiones fundamentalmente en el año 2004 (Islas Baleares, Castilla La Mancha, Madrid, Murcia y Navarra). Finalmente, la mayoría de las CC.AA. (Andalucía, Aragón, Castilla-León, Cataluña, Comunidad Valenciana, Galicia) realizaron sus inversiones más recientemente (año 2009). Es de destacar el importante esfuerzo económico realizado en las comunidades de Valencia y sobre todo de Cataluña. Dado que en el año 2009, la mayoría de las aglomeraciones urbanas tanto en Cataluña como en Valencia ya disponía de acceso a servicios de saneamiento, en ausencia de datos más actualizados, es de prever que dichas inversiones han tenido como objetivo la implementación de sistemas de tratamiento avanzados destinados a la reutilización del agua residual.

Figura 15. Evolución de las inversiones en tratamiento de aguas residuales por regiones españolas

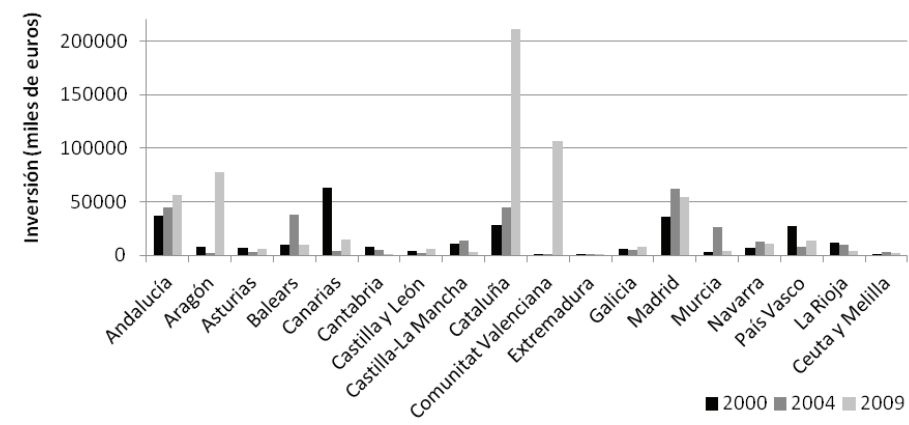

Fuente: Elaboración propia a partir de INE (2012) 


\section{CONCLUSIONES}

El acceso a los servicios básicos de saneamiento y depuración de aguas residuales es un aspecto de suma importancia tanto desde el punto de vista ambiental como sanitario. Así, los primitivos sistemas de alcantarillado desarrollados por el Imperio Mesopotámico, la Civilización Hindú y sobre todo la Civilización Griega y el Imperio Romano pusieron de manifiesto la importancia de la adecuada gestión del agua residual. Sin embargo, el final del Imperio Romano marcó el comienzo de los años negros del saneamiento en los que durante más de 1.000 años, el agua se vertía directamente sin ningún tipo de tratamiento constituyendo un importante foco de enfermedades. No obstante, a comienzos del siglo XIX está perspectiva cambió comenzando el resurgir del saneamiento si bien no fue hasta el siglo XX cuando tuvo lugar la gran revolución en el ámbito del saneamiento y depuración de aguas residuales.

A pesar de los importantes esfuerzos realizados, a nivel global todavía hay más de 2.600 millones de personas sin acceso a los servicios básicos de saneamiento. La mayoría de estas personas viven en el Sureste asiático, en el Este de Asia y África Subsahariana. Así mismo, existe una importante disparidad entre las áreas rurales y urbanas ya que 7 de cada 10 personas sin acceso al saneamiento viven en áreas rurales.

A nivel europeo, la Directiva 91/271/CEE supuso una importante mejora en el saneamiento y depuración de las aguas residuales en los Estados Miembro de la Unión Europea. Así, el 93\% de la carga contaminante generada en la UE-18 es recogida con sistemas de alcantarillado adecuados y el $87 \%$ es depurada con tratamientos de tipo de secundario. En relación a las zonas sensibles a la eutrofización, los distintos países europeos han adoptado diversas estrategias identificándose el 68\% del territorio de la UE-27 como sensible a la eutrofización. En este contexto, merece la pena destacar que las diferencias entre países europeos no sólo se producen en cuanto al porcentaje de agua depurada sino también en relación al grado de tratamiento recibido.

Nuestro país como miembro de la Unión Europea debe cumplir los requerimientos establecidos por la Directiva 91/271/CEE. Así, en el año 2006, el 77\% de las aglomeraciones urbanas mayores de 15.000 habitantes equivalentes estaban conformes a la Directiva. En este sentido, desde el año 2000 al 2009 se ha producido un aumento de más del $60 \%$ del volumen de agua residual tratada en España. Por otra parte, la reutilización del agua residual regenerada ha adquirido especial relevancia en las regiones españolas deficitarias de recursos hídricos, principalmente Murcia, Islas Baleares y Valencia. Este recurso no convencional es utilizado fundamentalmente para uso agrícola. Así mismo, merece la pena destacar el importante esfuerzo realizado por las Comunidades Autónomas en materia de saneamiento y depuración de aguas residuales ya que desde el año 2000 al 2009 se han invertido más de 3.700 millones de euros en esta materia. 


\section{BIBLIOGRAFÍA}

AIELLO, A.E., LARSON, M.S. y SEDLAK, R. (2008). Hidden heroes of the health revolution. Sanitation and personal hygiene. American Journal Infection Control, $36,128-151$.

COOPER, P.F. (2007). Historical aspects of wastewater treatment. In: Lens, P., Seeman, G., Lettinga, G. (eds). Decentralised sanitation and reuse: concepts, systems and implementation. IWA Publishing.

CUMMING, O. (2009). The sanitation imperative: A strategic response to a development crisis. Desalination, 248, 8 - 13.

GIOVANNINI, C. (1996). Rehabilitate the city. The utopia of the late nineteenth century. (In Italian), Geografia Umana. Milano: Franco Angeli Editore.

INE (Instituto Nacional de Estadística) (2012) Disponible en: http://www.ine.es/jaxi/ menu.do?type $=$ pcaxis \&path $=\% 2 \mathrm{Ft} 26 \% 2 \mathrm{Fp} 067 \% 2 \mathrm{Fp} 01 \&$ file $=$ inebase $\& \mathrm{~L}=($ Último acceso en 15 Febrero 2012).

JONES, D.E. (1967). Urban hydrology-a redirection. Civil Enginering, 37, 58 - 62.

LOFRANO, G. y BROWN, J. (2010). Wastewater management through the ages: A history of mankind, Science of the Total Environment, 408, 5254-5264.

MARM (Ministerio de Medio Ambiente, Rural y Marino) (2006). Plan Nacional de Calidad del Agua: Tratamiento de aguas residuales. Disponible en: http://www. mma.es/secciones/medios_comunicacion/prensa/notas_pre/2007/06/PlanNacionalCalidadAguas.pdf (Último acceso en 15 Febrero 2012).

SEEGER, H. (1999). The history of German wastewater treatment. European Water Management, 2, 51 - 56.

SIA (Sistema Integrado de Información del Agua) (2011) Disponible en: http://www. marm.es/es/agua/temas/sia/ (Último acceso en 15 Enero 2012).

SORI, E. (2001). The city and waste - Urban ecology from the Middle Ages to the early twentieth century. (In Italian). Saggi,Bologna: Il Mulino.

TARLOW, S. (2007). The archaeology of improvement in Britain, 1750-1850. Cambridge University Press.

TOLLE-KASTENBEIN R. (2005). Water Arqueology (In Italian). Longanesi.

UNEP (United Nations Environment Programme) (2010). Sick water? The central role of wastewater management in sustainable development. Birkeland Trykkery AS, Norwey.

WOLFE, P. (1999). History of wastewater. World of water 2000-the past, present and future. Water World/ Wastewater International Supplement to Penn Well Magazines, Tulsa, OH, USA. 
WORLD BANK (2006). Disease Control Priorities in Developing Countries, 2nd ed. WHO-UNICEF (World Health Organization-The United Nations Children's Fund) (2010). Progress Sanitation and Drinking-Water 2010 update. Disponible en: http://www.who.int/water_sanitation_health/publications/9789241563956/en/index.html (Último acceso en 15 Febrero 2012).

WISE (Water Information System for Europe, Sistema Europeo de acceso a información en material de agua) (2011). Disponible en: http://www.water.europa.eu/ (Último acceso en 15 Febrero 2012). 